\title{
Letter to the Editor: Surgery-Related Complications and Sequelae in Management of Tuberculosis of Spine
}

\author{
Myung-Sang Moon \\ Department of Orthopedic Surgery, Cheju Halla General Hospital, Jeju, Korea
}

I extend my appreciation to a reader of Asian Spine J who submitted "letter to the editor" regarding my article entitled "Surgery-related Complications and Sequelae in Management of Tuberculosis of Spine". For the issue raised by the reader, I would like to respond faithfully based on my clinical experiences in managing spinal tuberculosis for 60 years.

It is true that management of any disease evolves. Many surgeons have introduced their management experiences to journals. Frankly speaking, spinal tuberculosis is purely a medical condition because it is mycobacterial infection of the bony spinal column. Surgical intervention is indicated only when the spinal tuberculosis is complicated by disease progress or by clinically unacceptable (esthetically) degree of spinal deformity associated with or without neurological deficits.

It is important to define complicated spinal tuberculosis. Up to date, there is no internationally agreed and/or accepted definition and demarcation for complicated spinal tuberculosis. A few has defined the acceptable severity of kyphosis. Some patients will accept mild residual kyphosis $\left(<30^{\circ}\right)$, while others do not. Each individual has different view on the spinal cosmesis if his or her spinal column function is not impaired. Young patients, par- ticularly ladies, have high aesthetic demands, whereas the elderly has less demands on that.

In the early era (pre- and early chemotherapy era), the primary objective of management for spinal tuberculosis was to save life. However, in the later era (chemotherapy era), the primary objective was changed from life saving to deformity preventions and/or correction together with decompression surgery under the cover of anti-tuberculous chemotherapy.

In my practice before initiation of treatment, informed consent was routinely obtained from patients and their custodians. Residual kyphosis, management-related complications, and neural recovery rates according to the type of management were explained. Also, their esthetic demand related to residual kyphosis and agreement sign were obtained. Aesthetic demand was luxurious in the 1960s and early 1970s.

In the early era, four types of management were provided: 1) ambulatory chemotherapy alone; 2) anterior radical surgery \pm posterior fusion; 3 ) posterior fusion alone; 4) decompression surgery (costotransversectomy) by 1980 . The era of posterior instrumentation surgery began since early 1981.

Paraplegia was managed by two means: 1) conservative

Received Dec 1, 2014; Accepted Dec 2, 2014

Corresponding author: Myung-Sang Moon

Department of Orthopedic Surgery, Cheju Halla General Hospital, 65 Doryeong-ro, Jeju 690-766, Korea

Tel: +82-64-740-5030, Fax: +82-64-743-3110, E-mail: msmoonos@hotmail.com 
chemotherapy alone; 2) surgical decompression (radical surgery or costotransversectomy). In the late 1990s, it was found that posterior instrumented stabilization alone of the diseased segment hastened the neural recovery in a few days without direct decompression.

As regard to tuberculous kyphosis, there are two types of early collapse pattern: 1) kyphotic collapse of dorsal and D-L junction lesions; 2) telescoping one of the lumbar spine. In predicting residual kyphosis of conservatively treated patients, Rajasekaran and Shanmugasundaram's [1] formula was used since 1987 to explain the residual kyphoisis in patients $[2,3]$. This formula is helpful in estimating the fate of complicated spinal tuberculosis. Although this formula could be applicable in dorsal and D-L lesion, it was not good for lumbar lesion.

Oguz et al. [4] dealt with the advanced spinal tuberculosis-the "so-called complicated spinal tuberculos in adults". Their study had no control group [4]. Their management guideline could not be acceptable as the standard. Some may follow their recommendation. They presented illustrative cases in staging of the management. However, lesion sites were different. Type I was lumbar tuberculosis, but type II and type III were thoracolumbar tuberculosis. Clinical application of their classification has not yet been explored fully. They used 76 patients, which was not a huge case series. They stressed to treat spinal tuberculosis by surgery. Surgery can prevent and/ or correct the deformity and hasten neural recovery, but it can not bring early disease cure. They provided their own guideline in managing complicated spinal tuberculosis.

Up to now, no one has reported that they could shorten the chemotherapy period by surgery. Surgery has merits and demerits. No one has compared the cost-effectiveness of surgery with non-surgical management. British Medical Research Council study is not the obsolete one, and we still learn from it. Infected spines in children and adults take somewhat different clinical courses. Pedicle screw and hook systems affect the instrumented pediatric spine differently. In fact, I am the first one who introduced the posterior instrumentation surgery in managing spinal tuberculosis in the late 1980s.

Anterior radical surgery in pediatric spine sacrifices growth plates. For this issue, there were two different opinions: Upadhyay et al. $(1994,1996)$ [5] reported that anterior column growth was not affected by radical surgery, whereas Schulitz et al. [6] in 1997 reported that growth was affected. I do not prefer to use cage in ante- rior fusion surgery. Tuli in India stressed that residual kyphosis over $60^{\circ}$ should not be allowed because those patients with residual kyphosis $>60^{\circ}$ developed gradual paralysis [2,3,7-10].

Regarding spinal deformity, individual (poor or rich, young or old) has different aesthetic demands. Nowadays in Korea, since the introduction of nationwide medical insurance scheme, any citizen can knock on the door of any clinic any day any time. Therefore, patient with spinal diseases including spinal infections can be found in the early disease stage for which successful conservative treatment can be given. The diagnosis of spinal tuberculosis can only be delayed in immuno-compromised patients. Effective running of the health care system revolutionized the patterns of clinical practices.

Unfortunately, it was found that patients in under-privileged countries and communities had late management opportunities, although they had complicated spinal tuberculosis. All those patients were surgical care candidates.

Dr. Ito [11] in Kyoto, Japan who performed the anterior surgery for tuberculosis of spine in 1934 failed to cure the disease (pre-chemotherapy era) [9]. However, in the late 1950s, Dr. Hodgson and Dr. Stock [12] in Hong Kong conducted the same anterior surgery under the cover of chemotherapy with successful outcome in curing the disease. The different outcome in two series was due to antituberculous medications. Anti-tuberculous drugs cured the disease. Surgery contributed some in the correction and/or prevention of deformity. This fact clearly clarifies that spinal tuberculosis is primarily a medical condition. Honestly speaking, Hodgson was a lucky person indeed to be known as a great master surgeon thanks to anti-tuberculous medications. Now we are in the era of minimally invasive surgery. Therefore, we should apply minimum invasive surgery in our spinal practices.

Lastly I admit that Oguz et al. contributed to the promotion of surgical management for spinal tuberculosis. However, their guideline should not be used as bible. We should not try to solve clinical problems that we encounter using only one approach. Surgeons should meet the patients' complaints. I thank the reader for submitting "letter of the editor" related to my article. I hope that my response will be helpful in his practice.

\section{Conflict of Interest}

No potential conflict of interest relevant to this article 
was reported.

\section{References}

1. Rajasekaran S, Shanmugasundaram TK. Prediction of the angle of gibbus deformity in tuberculosis of the spine. J Bone Joint Surg Am 1987;69:503-9.

2. Moon MS, Kim SS, Moon H. (i) Tuberculosis of the spine: current views in diagnosis, management, and setting a global standard. Orthop Trauma 2013;27:185-94.

3. Moon MS. Tuberculosis of spine: current views in diagnosis and management. Asian Spine J 2014;8:97111.

4. Oguz E, Sehirlioglu A, Altinmakas M, et al. A new classification and guide for surgical treatment of spinal tuberculosis. Int Orthop 2008;32:127-33.

5. Upadhyay SS, Saji MJ, Sell P, Sell B, Hsu LC. Spinal deformity after childhood surgery for tuberculosis of the spine. A comparison of radical surgery and debridement. J Bone Joint Surg Br 1994;76:91-8.
6. Schulitz KP, Kothe R, Leong JC, Wehling P. Growth changes of solidly fused kyphotic bloc after surgery for tuberculosis. Comparison of four procedures. Spine (Phila Pa 1976) 1997;22:1150-5.

7. Moon MS. Development in the management of tuberculosis of the spine. Curr Orthop 2006;20:132-40.

8. Moon MS. Tuberculosis of spine-Contemporary thoughts on current issues and perspective views. Curr Orthop 2007;21:364-79.

9. Moon MS, Kim SS, Lee BJ, Lin JF. Tuberculosis of the spine: evolution in diagnosis and management. ArgoSpine News J 2011;23:90-8.

10. Tuli SM. Severe kyphotic deformity in tuberculosis of the spine. Int Orthop 1995;19:327-31.

11. Ito H, Suchiya J, Asami G. A new radical operation for Pott's disease: report of ten cases. J Bone Joint Surg Am 1934;16:499-515.

12. Hodgson AR, Stock FE. A preliminary communication on radical treatment of Pott's disease and Pott's paraplegia. Br J Surg 1956;44:266-75. 